\title{
Formation and transformation of vortex structures in soft ferromagnetic ellipsoids
}

A. S. Arrott, and R. Hertel

Citation: Journal of Applied Physics 103, 07 E739 (2008);

View online: https://doi.org/10.1063/1.2837499

View Table of Contents: http://aip.scitation.org/toc/jap/103/7

Published by the American Institute of Physics

\section{Articles you may be interested in}

The design and verification of MuMax3

AIP Advances 4, 107133 (2014); 10.1063/1.4899186

Current controlled random-access memory based on magnetic vortex handedness

Applied Physics Letters 93, 142508 (2008); 10.1063/1.2998584

Reliable low-power control of ultrafast vortex-core switching with the selectivity in an array of vortex states by inplane circular-rotational magnetic fields and spin-polarized currents

Applied Physics Letters 92, 022509 (2008); 10.1063/1.2807274

Applications of the gyrocoupling vector and dissipation dyadic in the dynamics of magnetic domains Journal of Applied Physics 45, 377 (2003); 10.1063/1.1662989

Chirality control of magnetic vortex in a square Py dot using current-induced Oersted field Applied Physics Letters 99, 242507 (2011); 10.1063/1.3669410

Dynamics of vortex core switching in ferromagnetic nanodisks

Applied Physics Letters 89, 262507 (2006); 10.1063/1.2424673

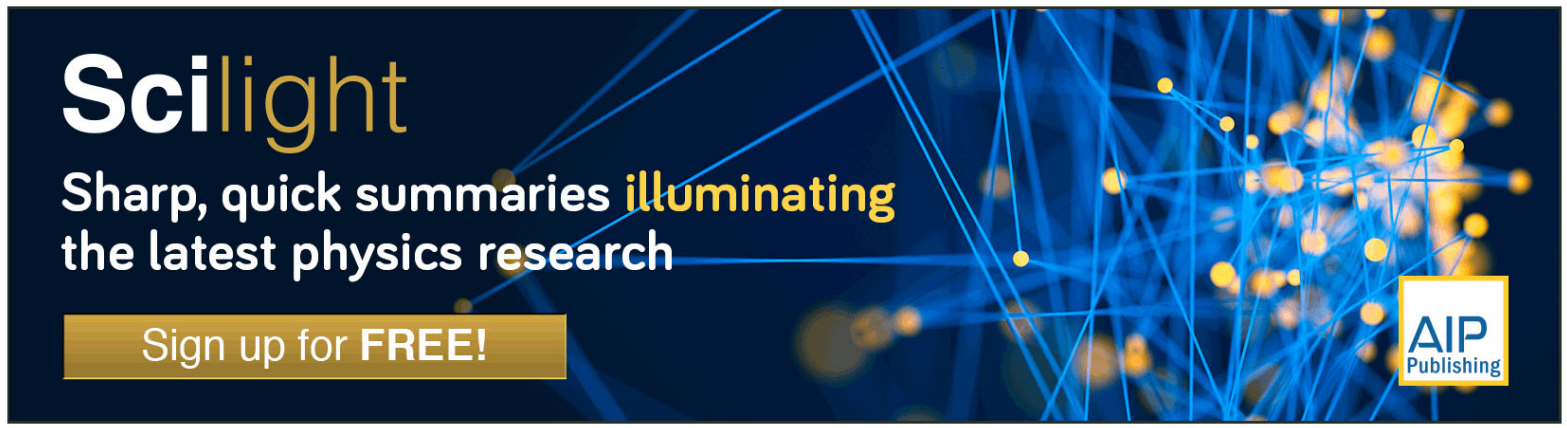




\title{
Formation and transformation of vortex structures in soft ferromagnetic ellipsoids
}

\author{
A. S. Arrott ${ }^{1, a)}$ and R. Hertel ${ }^{2}$ \\ ${ }^{1}$ Department of Physics, Simon Fraser University, Burnaby, British Columbia V5A 1S6, Canada \\ ${ }^{2}$ Institute of Solid State Research IFF-9, Research Center Jülich, Jülich, Germany
}

(Presented on 6 November 2007; received 12 September 2007; accepted 29 November 2007; published online 25 March 2008)

\begin{abstract}
Magnetization processes in ideally soft ferromagnetic ellipsoids are studied numerically using the equations of micromagnetism. The ellipsoids have semiaxis in the ratios $b / a=c / b=\phi$, where $\phi$ is the golden ratio for which $(c-b) / a=1$. For $a>20 \mathrm{~nm}$, the magnetization processes include the formation and transformation of a vortex core. As vortex core motion takes place with little change in exchange energy, the response of the total magnetization to the applied fields is close to that of an ideally soft magnetic material as long as the vortex is mobile. At critical fields, the vortex core changes between states where it is free to move and those where it is immobilized by symmetry. Complex transformations at critical fields are observed. () 2008 American Institute of Physics.
\end{abstract} [DOI: $10.1063 / 1.2837499]$

In soft ferromagnetic materials with dimensions that are all larger than the characteristic exchange length, but not too much larger, the "vortex core" plays an important role in the magnetization process. We study, using numerical methods of both finite element and finite grid, the Landau-LifshitzGilbert equations ${ }^{1}$ as applied to ellipsoids with three unequal axes in response to series of magnetic fields, uniform and nonuniform, applied along the principal axes of the ellipsoids. The semiaxes, $a, b$, and $c$, are chosen using the golden ratio: $b / a=c / b=\phi=1.6180339 \cdots$, for which $(c-b) / a=1$. Here, this shape is called the $\phi$ ellipsoid.

Above a threshold size, $a>20 \mathrm{~nm}$, the pattern assumed in zero field, regardless of the directions of the previous fields, is a stable configuration that is definitely not uniform (see Figs. 1(a)-1(d), the insets in Fig. 2, and the detailed discussion of the graphics in the figure captions). This vortex core structure undergoes transformations and reformations during the magnetization processes in applied fields. Large localized magnetic fields are produced just outside the ellipsoid, where the core intersects the surface. These have potential uses in information technology.

The vortex core in the ellipsoid has a direct correspondence to the Bloch wall with its Nèel cap, as observed and calculated for the Landau structure in an iron whisker ${ }^{1}$ and in mesoscopic iron particles. ${ }^{2}$ An iron whisker has crystalline anisotropy and magnetostriction, but these are secondary effects, which are neglected in these calculations. In this approximation, the equations of motion are readily expressed in reduced units $h=\left(\mu_{0} H\right) /\left(\mu_{0} M_{s}\right), m=M / M_{s}$, and $x^{\prime}=x / a$ with the results dependent on the single parameter $\Lambda=\lambda / a$, where $\lambda=\left(0.5 \mathrm{~A} / \mu_{0}\right)^{1 / 2} M_{s}$ is the magnetostatic exchange length. In this work, we use unreduced units for Permalloy, where $\mu_{0} M_{s}=1 \mathrm{~T}$ and $\lambda=5 \mathrm{~nm}$.

When the field is reduced from a value sufficient for saturation, the magnetization goes first to the curling state,

${ }^{a)}$ Electronic mail: arrott@sfu.ca. which has a sense of rotation about the axis of the applied field. If the pattern maintains inversion symmetry, the sense of rotation is opposite on either sides of the perpendicular midplane, leading to a "Nèel wall" there. ${ }^{3}$ This is avoided by forcing the nucleation of a single sense of rotation by applying a current along the axis of the field. ${ }^{4}$ The current can be removed after nucleation of the curling pattern. Its effect is readily taken into account analytically.

In the curling pattern, the magnetization turns away from the field axis more rapidly with distance from that axis along the shorter of the two perpendicular axes. As a result, the pattern resembles a Bloch wall from one end to the other of the shorter axis. However, the main effect is that the curling pattern has a vortex core that is forced by the symmetry of the ellipsoid in the applied field. The magnetization reversal

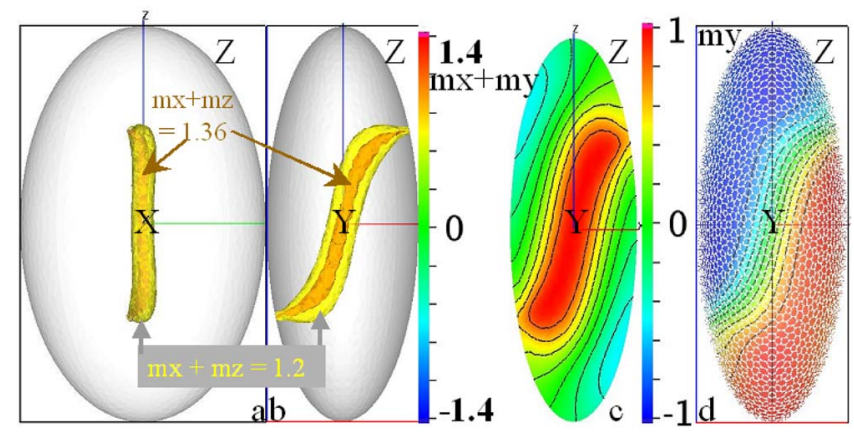

FIG. 1. (Color online) The principal states of an ideally soft ferromagnetic $\phi$-ellipsoid with $a=100 \mathrm{~nm}$. The panels a-d show the vortex configuration in zero field. For $a$ and $b$ the ellipsoid surface is semitransparent as is the isosurface for $m z+m x=1.2$, which is the locus of all moments that make an angle of $30^{\circ}$ with the $(1,0,1)$ direction. The inner core corresponds to $12.5^{\circ}$ from the $(1,0,1)$. In $\mathrm{c}$ and $\mathrm{d}$ the vortex is appears in the contour plots on the midplane with $y=0$. The contours for $m z+m x$ in c correspond to b. As the vortex lies close to a plane of constant $y$ and has its magnetic components in that plane, it appears in (d) as the green contour for $m y=0$. The mesh used in the finite element calculation is displayed in $d$, where it intersects the semitransparent ellipsoid surface. The finite element simulation results are visualized with GMV. 


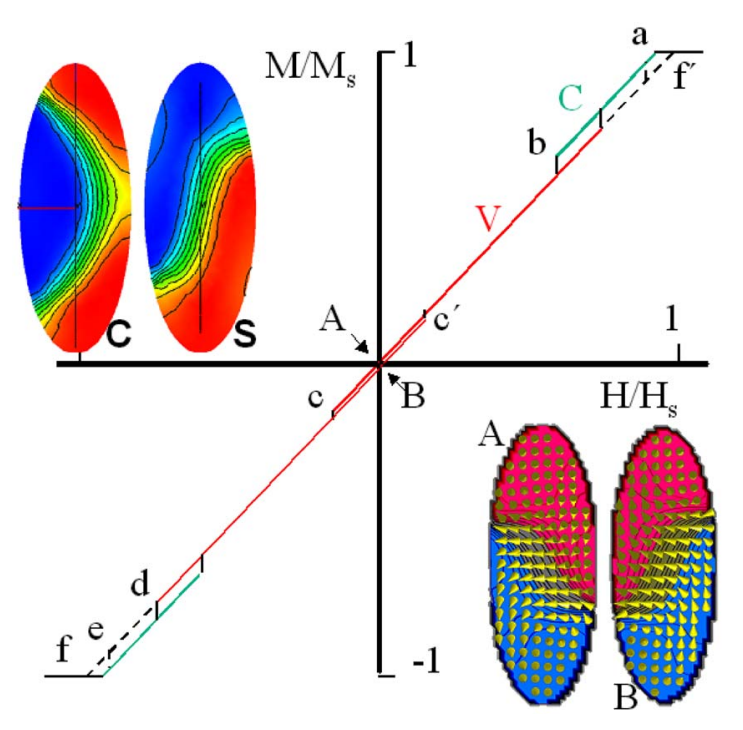

FIG. 2. (Color online) An idealization of the processes for magnetization $M$ in a $\phi$-ellipsoid large enough to sustain a single vortex core. On reducing the field $H$ from saturation, the initial process is one of curling with the handedness selected by a small current along the axis of the field. In the curling state, labeled $\mathbf{C}$ on the hysteresis loop, the exchange energy is proportional to the decrease in the magnetization along the field. This yields a constant effective exchange field that aids the applied field in maintaining a net magnetization. Below a critical field labeled $b$, the vortex core breaks free from the axis of the field. In the V-state the "free vortex core" moves with little change in exchange energy, following close to the behavior of a magnetically soft ellipsoid. There is a small remanence from the magnetization of the core, indicated by the state A. At c, the core switches from state A to state $\mathrm{B}$, which are shown in the lower right quadrant as they appear in zero field. (The external field where the vortex intersects the surface is $\mu_{0} H$ $=0.4 \mathrm{~T}$ close to the surface, decreasing to $0.1 \mathrm{~T}$ at $10 \mathrm{~nm}$ from the surface For the $\phi$-ellipsoid with shortest minor axis $a=50 \mathrm{~nm}$, the switching field is $0.03 \mathrm{~T}$. The cones represent the mean direction of a large number of cells as the grid is $2 \mathrm{~nm}$. The calculations using finite grids do not have smooth surfaces.) At somewhere between $\mathrm{d}$ and $\mathrm{e}$, the vortex may jump into the curling state, or else it might go to saturation continuously at $\mathrm{f}$, depending on size. Between $\mathrm{f}^{\prime}$ and a, the magnetization is not fully saturated. The transitions at $\mathrm{b}$ are the most complicated part of the magnetization processes. For large $\phi$-ellipsoids the transition takes place in two stages at two different fields, one of which goes from the curling state to a vortex state with a $\mathrm{C}$-shaped profile and the other goes from the $\mathrm{C}$-shaped profile to the $\mathrm{S}$-shaped profile. The $\mathrm{S}$-shaped profile never goes to the $\mathrm{C}$-shaped profile. The $\mathrm{C}$ and $\mathrm{S}$ states are shown as contours of $m y$, from +1 (red) to -1 (blue) in the $y=0$ mid-plane. The escape of the vortex from the curling state about the $\mathrm{z}$-axis can be into either a $\mathrm{C}$ state or an $\mathrm{S}$ state. The core of the vortex is indicated by the green area where $m y$ is zero. When the escape is to the $\mathrm{C}$ state, it is then necessary for the vortex to switch to the S state, which is accomplished by the upper end of the vortex core sweeping over the north pole.

must do something with that core. One possibility is the propagation down its axis of point singularities formed at the surface. Another possibility is the formation of pairs of singularities somewhere along its axis, which then propagate to reverse the core. ${ }^{3,5}$ Usually, the vortex finds freedom by breaking away from the axis of symmetry. The way in which this happens is different for fields along each of the axes of symmetry and can be quite complex. As an example, we show in Fig. 2, two configurations labeled as $\mathrm{C}$ and $\mathrm{S}$, which can occur as the vortex breaks away from the $z$ axis upon reducing the field $H_{z}$, depending on how the symmetry is broken. If the $\mathrm{C}$ state is formed, it must transform to the $\mathrm{S}$ state in order to make the transition from state A to state B (see Fig. 2). It does this by passing one arm of the $\mathrm{C}$ state over the north pole in a dynamic process at a critical field. That critical field can be the same as the nucleation field $b$ or it can approach zero field depending on the size of the $\phi$ ellipsoid.

To describe the behavior with increasing field in the $z$ direction, starting from zero field with the configuration of Figs. 1(a)-1(d), is not difficult because the vortex mainly lies parallel to the $y=0$ plane and gradually aligns along the field axis. The vortex core, which is stable in zero field for $a$ $>20 \mathrm{~nm}$, has four possible states, two of which are shown in Fig. 2 and labeled as A and B. In A, the vortex core intersects the surface at $\left(-x_{c}, y_{c},-z_{c}\right)$ and $\left(x_{c}, y_{c}, z_{c}\right)$. In $\mathrm{B}$, the intersections are at $\left(-x_{c}, y_{c}, z_{c}\right)$ and $\left(x_{c}, y_{c},-z_{c}\right)$. For each of these, the magnetization could be in the opposite direction. The direction of the offset $y_{c}$ is not arbitrary as $y_{c}$ and $-y_{c}$ have different energies for each of the four states. The only difference in magnetic response comes from the direction of the core magnetization with respect to the applied field. If it is opposite to the applied field, the vortex core can flip from state A to state $\mathrm{B}$. The magnetization process involving the flip is shown in Fig. 2. The position of the vortex at the surface can be moved through a finite distance using a pulsed critical field. The field outside the vortex $\mu_{0} H_{\text {ext }}$ is of the order of $\mu_{0} M_{s} / 2$. This highly localized large field may be useful in devices. Once the vortex has flipped from state B to state $\mathrm{A}$, it follows the same magnetization process as if it had started in state A.

From extensive studies of the magnetization processes for different axes and sizes ranging from $a=10$ to $100 \mathrm{~nm}$, it is possible to make some general remarks about the hysteresis loops of ideally soft $\phi$ ellipsoids. In the limit of negligible magnetocrystalline anisotropy and the absence of magnetoelastic effects, the magnetization process is a competition among the three energy terms, magnetostatic self-energy, the Zeeman energy of the magnetization in the applied fields, and the increase in exchange energy that accompanies nonuniform magnetization. As the applied field is reduced from a value sufficient to saturate the magnetization, the onset of the curling pattern leads to an increase in the total exchange energy that is proportional to the decrease in the total magnetization along the field. The derivative of the total exchange energy with respect to the total magnetization can be considered to be an effective field. That field is a constant during the onset of curling and shifts the magnetization curve, whose slope is determined by the demagnetizing factor for the ellipsoid along the field axis, to lower fields. This is seen schematically in the idealized magnetization curve of Fig. 2.

The competition between the magnetostatic energy and the applied field in the absence of an exchange field leads to a linear relation between field and magnetization with the slope being determined by the demagnetizing factor for the axis of the field. This sets the scale for the application of Fig. 2 to each of the results. This differs from the case of rotation in unison, where it is the difference between the two demagnetizing factors in the plane of rotation that matters. Our results are in agreement with the classical demagnetizing factors for uniformly magnetized ellipsoids, ${ }^{6}$ even with the highly nonuniform magnetization patterns. 
The effect of the constant field from the linear variation of the exchange energy with magnetization is to offset the hysteresis loop, as shown in Fig. 2, as long as the curling pattern persists. The micromagnetic calculations yield the coefficient of this linear dependence for each direction of the magnetic field. It becomes increasingly important as the size of the $\phi$ ellipsoid decreases. Once the vortex breaks free, the magnetization process takes place with much smaller change in exchange energy with magnetization. This is the same as the effect of a domain wall forming and moving in a whisker. The magnetization in the process of core motion is slightly offset in the direction of the magnetization of the core. The core reverses at $c$ and $c^{\prime}$ in Fig. 2.

In this idealization of the magnetization processes, the branches of the hysteresis loop in Fig. 2 are determined by the demagnetizing factor, the magnetization derivative of the exchange energy in the $C$ region, and the net moment of the vortex core in the $V$ region. How these factors determine the transition fields, labeled a, b, c, d, e, and f, are less obvious, even after the results of the computations are known.

The methodology used is based on our experience in micromagnetic calculations. We use bias fields ${ }^{4}$ and the path method $^{7}$ for determining critical fields with precision. Bias fields from currents are used to break symmetries. ${ }^{4}$ This is much like measuring the magnetization in a small field to determine the Curie temperature of a ferromagnet and then using Landau theory to correct for the effect of the small bias field. Equilibrium values are found using extrapolation techniques applied to magnetization components and energies. After sufficient time, the total energy approaches equilibrium precisely as a simple exponential. Then, the magnetization components and the individual energies are precisely fit to damped oscillators (we use $\alpha=0.5$ for the damping coefficient). Our results are converged when the extrapolated values no longer change with time within a prescribed precision.

As our extensive treatment of the $\phi$ ellipsoid for $a$ between 20 and $200 \mathrm{~nm}$ with fields and currents applied along each of the three principle axes cannot fit in this limited note, we can only promise to try to present these results elsewhere where the movies can be stored with the archival material. The distorted vortex core is best seen by rotating it, as is possible in three-dimensional graphics programs. ${ }^{8}$

${ }^{1}$ A. Hubert and R. Schäfer, Magnetic Domains: The Analysis of Magnetic Microstructures (Springer, Berlin, 1998), Chap. 3.

${ }^{2}$ R. Hertel, O. Fruchart, S. Cherifi, P.-O. Jubert, S. Heun, A. Locatelli, and J. Kirschner, Phys. Rev. B 72, 214409 (2005).

${ }^{3}$ A. S. Arrott, J. Magn. Magn. Mater. 215-216, 6 (2000).

${ }^{4}$ A. S. Arrott and R. Hertel, IEEE Trans. Magn. 43, 2911 (2007).

${ }^{5}$ R. Hertel and J. Kirschner, J. Magn. Magn. Mater. 278, L291 (2004).

${ }^{6}$ J. A. Osborn, Phys. Rev. 67, 351 (1945).

${ }^{7}$ M. R. Scheinfein and A. S. Arrott, J. Appl. Phys. 93, 6802 (2003).

${ }^{8}$ See http://www-xdiv.lanl.gov/XCM/gmv/GMVHome.html. 